\title{
Insight about funding, communication and data sharing for early-career scientists
}

\author{
Niina Kuosmanen', E.J. Gowan² and K. Braun ${ }^{3}$
}

pointed out that the promise of excellence means highlighting the potential of making a breakthrough in your field of research.

The same topic was then also discussed within breakout groups. The participants underlined the importance of time management between research and grant application. Indeed, you might have to write several proposals before one is selected. Don't lose patience! On the contrary, take advantage of any review received, and even ask for independent reviews to improve your proposal. Starting from a small proposal with welldefined goals and objectives and letting it evolve towards a broader research project was also a strategy that the breakout groups proposed.

\section{Workshop 2: How to communicate science} Communicating science covers many aspects from writing a research proposal to making your research understood by people who are not familiar with your field. Alicia Newton (Nature Geoscience) stressed a few questions to successfully communicate research in a focused manner: Which specific question do you want to answer? What is the purpose of your research? Why should people believe you? Knowing your audience and adapting your message to it are also crucial to be understood.

Liz Kalaugher (environmentalresearchweb) spoke about promoting your research to the media. Making your research known

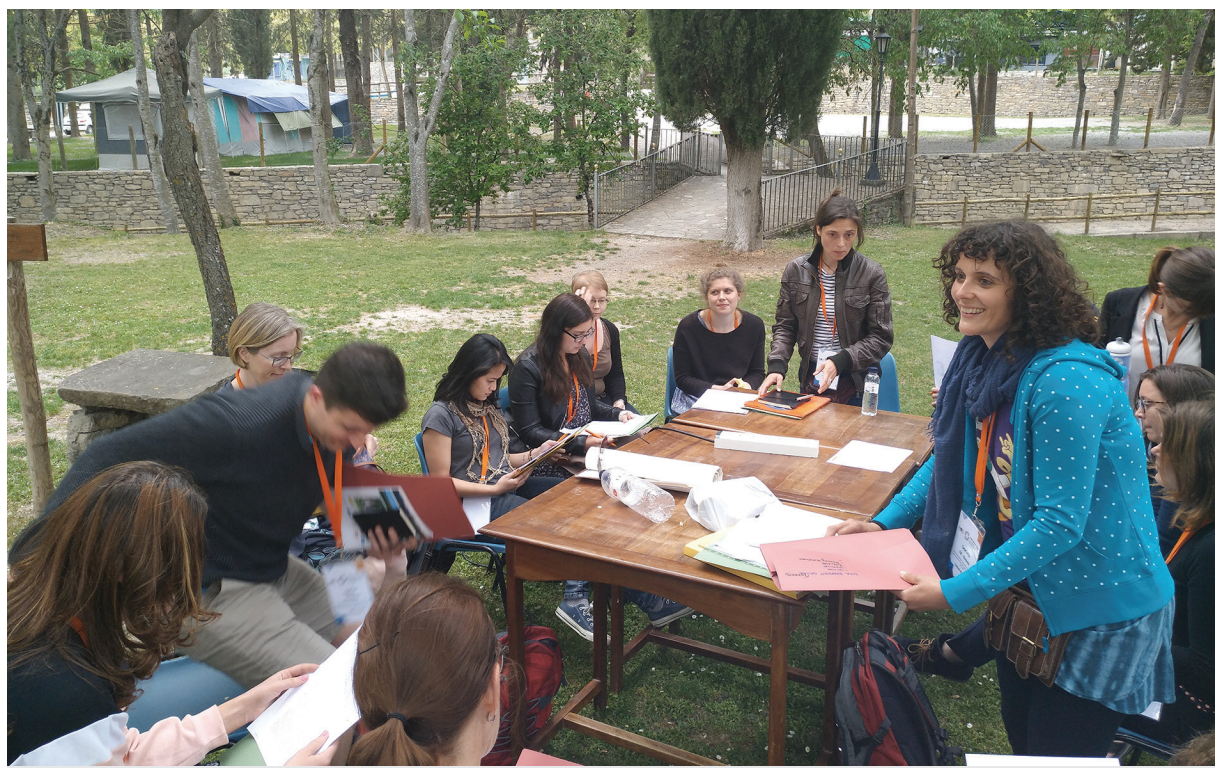

Graciela Gil-Romera, a member of the YSM Local Organizing Committee, hands out a list of possible questions for discussion at the "Communication" round-table meeting

and being involved in outreach outside of your own research community can raise your profile and may result in more citations. However, not every study makes it to the news. Studies directly affecting people's lives and controversial and unusual topics are most likely to get attention. Nevertheless, active promotion of your research in blogs, social media or at outreach events may increase your media exposure.

Angela Wade (PAGES) introduced the possibilities for early-career scientists to use publishing and other communication outlets within the PAGES network. One important conclusion from the discussion was the demand for more extensive workshops on science communication for early-career scientists.

\section{Workshop 3: Data sharing}

In the third workshop Darrell Kaufman (Northern Arizona University), Alicia Newton and Jonas Bunikis discussed the opportunities and challenges of sharing data. Making data freely and broadly accessible is important, because large data sets allow for questions to be tackled that cannot be answered by a single study. Data sets, even small ones, provide the means for assessing spatio-temporal patterns, conducting proxy-proxy comparisons and data-model comparisons, and identifying aberrant records. Challenges in data sharing include the often heterogeneous and multidimensional data and nuanced interpretation of different data types. Having a data management plan early in your career is essential, since both journals and funding agencies increasingly require documentation of data availability. Publication in data-oriented journals provides description for the data sets, favors the sharing and reuse of data and gives credit to those who share their data. Safe repository (for example with a certified organization) offers long-term archives for your data and easy citation.

Altogether, these three workshops provided valuable knowledge, topics for further thought and discussion, and guidance for our scientific careers.

\section{AFFILIATIONS}

'Department of Forest Ecology, Czech University of Life Sciences Praque, Czech Republic

${ }^{2}$ Alfred Wegener Institute, Bremerhaven, Germany ${ }^{3}$ Institute of Human Origins, Arizona State University, Tempe, USA 